\title{
Some results for demimartingales and $N$-demimartingales
}

Pingping Dai, Yan Shen*, Shuhe Hu and Wenzhi Yang

"Correspondence:

shenyan@ahu.edu.cn

School of Mathematical Science, Anhui University, Hefei, 230039,

P.R. China

Full list of author information is

available at the end of the article

\begin{abstract}
In this paper, we obtain some results such as maximal and minimal type inequalities for demisubmartingales and demimartingales. Meanwhile, by giving an example, we point out that the Chow type maximal inequality of $\mathrm{N}$-demimartingales is not true, which affects some maximal type inequalities for $\mathrm{N}$-demimartingales.
\end{abstract}

MSC: 60E15; 60F15

Keywords: maximal inequality; demimartingales; $N$-demimartingales; minimal inequality

\section{Introduction}

Let $S_{1}, S_{2}, \ldots, S_{n}, \ldots$ be a sequence of random variables defined on a probability space $(\Omega, \mathcal{F}, P)$ and $S_{0}=0$.

Definition 1.1 Let $\left\{S_{j}, j \geq 1\right\}$ be an $L^{1}$ sequence of random variables. Assume that for $j=$ $1,2, \ldots$,

$$
E\left\{\left(S_{j+1}-S_{j}\right) f\left(S_{1}, \ldots, S_{j}\right)\right\} \geq 0
$$

for all coordinatewise nondecreasing functions $f$ such that the expectation is defined. Then $\left\{S_{j}, j \geq 1\right\}$ is called a demimartingale. If in addition the function $f$ is assumed to be nonnegative, the sequence $\left\{S_{j}, j \geq 1\right\}$ is called a demisubmartingale.

Definition 1.2 Let $\left\{S_{j}, j \geq 1\right\}$ be an $L^{1}$ sequence of random variables. Assume that for $j=1,2, \ldots$,

$$
E\left\{\left(S_{j+1}-S_{j}\right) f\left(S_{1}, \ldots, S_{j}\right)\right\} \leq 0
$$

for all coordinatewise nondecreasing functions $f$ such that the expectation is defined. Then $\left\{S_{j}, j \geq 1\right\}$ is called an $N$-demimartingale. If in addition the function $f$ is assumed to be nonnegative, the sequence $\left\{S_{j}, j \geq 1\right\}$ is called an $N$-demisupermartingale.

The concepts of demimartingales and demisubmartingales were due to Newman and Wright [1]. It can be checked that a submartingale with the natural choice of $\sigma$ algebras is a demisubmartingale, but the converse statement cannot always be true. Newman and Wright [1] proved that the partial sums of mean zero associated random

(C2014 Dai et al.; licensee Springer. This is an Open Access article distributed under the terms of the Creative Commons Attribution License (http://creativecommons.org/licenses/by/2.0), which permits unrestricted use, distribution, and reproduction in any medium, provided the original work is properly cited. 
variables form a demimartingale. Similarly, the notion of $N$-demimartingales and $N$ demisupermartingales can be found in Christofides [2]. It is trivial to verify that the partial sums of mean zero negatively associated random variables form an $N$-demimartingale, and a supermartingale with the natural choice of $\sigma$-algebras is an $N$-demisupermartingale, but the converse statement cannot always be true (see Christofides [2]). Various results and examples of demisubmartingales and demimartingales have been obtained. For example, Newman and Wright [1] obtained Doob type maximal inequalities and upcrossing inequality for demisubmartingales; Wood [3] investigated more properties of demimartingales; Christofides [4] generalized the Chow type maximal inequalities for demisubmartingales; Prakasa Rao [5] investigated the Whittle type maximal inequality for demisubmartingales; Christofides [6] constructed some $U$-statistics based on associated random variables and proved them to be demimartingales; Wang [7] studied some maximal inequalities for associated random variables and demimartingales; Prakasa Rao [8] obtained more maximal and minimal type inequalities for demisubmartingales; Wang and $\mathrm{Hu}$ [9] also studied some maximal inequalities for demimartingales and their applications; Wang et al. [10] gave a Doob type inequality and a strong law of large numbers for demimartingales; Wang et al. [11] also studied the maximal and minimal type inequalities for demimartingales; Christofides and Hadjikyriakou [12] gave some maximal and moment inequalities for demimartingales; Hu et al. [13] investigated the Marshall type inequalities for demimartingales; Wang et al. [14] got some maximal inequalities for demimartingales based on concave Young functions. Meanwhile, for the results of $N$ demisupermartingales and $N$-demimartingales, Christofides [2] gave some maximal type inequalities for $N$-demimartingales; Prakasa Rao [15] studied the Chow type maximal inequality for $N$-demimartingales, Christofides and Hadjikyriakou [16] got some exponential inequalities for $\mathrm{N}$-demimartingales; Hu et al. [17] gave a note on the inequalities for $\mathrm{N}$ demimartingales; Hadjikyriakou [18] obtained a Marcinkiewicz-Zygmund type inequality for nonnegative $N$-demimartingales; Wang et al. [19] studied some maximal type inequalities for $N$-demimartingales and provided a strong law of large numbers as an application; Yang and $\mathrm{Hu}[20]$ investigated more maximal type inequalities for $\mathrm{N}$-demimartingales, etc. For more results and examples of demimartingales and $N$-demimartingales, one can refer to Prakasa Rao [21] and Hadjikyriakou [22]. On the other hand, the conditional demimartingales and $N$-demimartingales have received more attention; we refer to Christofides and Hadjikyriakou [23], Wang and Wang [24], Prakasa Rao [21] and Hadjikyriakou [22], etc.

Inspired by the papers above, we investigate some maximal and minimal type inequalities for demisubmartingales and demimartingales. Meanwhile, by giving an example, we point out that the Chow type maximal inequality of $N$-demimartingales is not true, which affects some maximal type inequalities for $N$-demimartingales.

Throughout this paper, let $I(A)$ denote the indicator function of the set $A$ and $x^{+}=I$ $(x \geq 0)$.

Lemma 1.1 (Christofides [4, Lemma 2.1]) Let $\left\{S_{n}, n \geq 1\right\}$ be a demisubmartingale (or a demimartingale) and $g$ be a nondecreasing convex function such that $g\left(S_{i}\right) \in L^{1}, i \geq 1$. Then $\left\{g\left(S_{n}\right), n \geq 1\right\}$ is a demisubmartingale.

\section{Main results}

First, we provide a maximal type inequality for a sequence of demisubmartingales. 
Theorem 2.1 Let $\left\{S_{n}, n \geq 1\right\}$ be a demisubmartingale with $S_{0}=0$ and assume that $\left\{c_{n}, n \geq\right.$ $1\}$ is a nondecreasing sequence of positive numbers. Then, for any $\varepsilon>0$,

$$
\varepsilon P\left\{\max _{1 \leq k \leq n} c_{k} S_{k} \geq \varepsilon\right\} \leq c_{n} E\left[S_{n}^{+} I\left(\max _{1 \leq k \leq n} c_{k} S_{k} \geq \varepsilon\right)\right] .
$$

Proof Following Christofides [4], we give the proof of Theorem 2.1. For fixed $n \geq 1$, let $A=\left\{\max _{1 \leq k \leq n} c_{k} S_{k} \geq \varepsilon\right\}$. Then $A$ can be written as $A=\bigcup_{j=1}^{n} A_{j}$, where $A_{1}=\left\{c_{1} S_{1} \geq \varepsilon\right\}$, $A_{j}=\left\{c_{i} S_{i}<\varepsilon, 1 \leq i<j, c_{j} S_{j} \geq \varepsilon\right\}, 1<j \leq n$, and $A_{i} \cap A_{j}=\emptyset$ when $i \neq j$. Therefore, one has

$$
\begin{aligned}
\varepsilon P(A) & =\varepsilon \sum_{j=1}^{n} P\left(A_{j}\right)=\sum_{j=1}^{n} E\left(\varepsilon I_{A_{j}}\right) \leq \sum_{j=1}^{n} E\left(c_{j} S_{j} I_{A_{j}}\right)=\sum_{j=1}^{n} E\left(c_{j} S_{j}^{+} I_{A_{j}}\right) \\
& =E\left(c_{1} S_{1}^{+} I_{A_{1}}\right)+E\left(c_{2} S_{2}^{+} I_{A_{2}}\right)+\sum_{j=3}^{n} E\left(c_{j} S_{j}^{+} I_{A_{j}}\right) \\
& =E\left(c_{1} S_{1}^{+} I_{A_{1}}\right)+E\left[c_{2} S_{2}^{+}\left(I_{A_{1} \cup A_{2}}-I_{A_{1}}\right)\right]+\sum_{j=3}^{n} E\left(c_{j} S_{j}^{+} I_{A_{j}}\right) \\
& =E\left(c_{2} S_{2}^{+} I_{A_{1} \cup A_{2}}\right)+E\left[\left(c_{1} S_{1}^{+}-c_{2} S_{2}^{+}\right) I_{A_{1}}\right]+\sum_{j=3}^{n} E\left(c_{j} S_{j}^{+} I_{A_{j}}\right) \\
& \leq E\left(c_{2} S_{2}^{+} I_{A_{1} \cup A_{2}}\right)+c_{2} E\left[\left(S_{1}^{+}-S_{2}^{+}\right) I_{A_{1}}\right]+\sum_{j=3}^{n} E\left(c_{j} S_{j}^{+} I_{A_{j}}\right) \\
& =E\left(c_{2} S_{2}^{+} I_{A_{1} \cup A_{2}}\right)-c_{2} E\left[\left(S_{2}^{+}-S_{1}^{+}\right) I_{A_{1}}\right]+\sum_{j=3}^{n} E\left(c_{j} S_{j}^{+} I_{A_{j}}\right),
\end{aligned}
$$

which is from the facts that $A_{1} \cap A_{2}=\emptyset, I_{A_{2}}=I_{A_{1} \cup A_{2}}-I_{A_{1}}$ and $\left\{c_{k}, k \geq 1\right\}$ is a nondecreasing sequence of positive numbers.

Let $h(y)=\lim _{x \rightarrow y^{-}}\left(x^{+}-y^{+}\right) /(x-y)$ and $f(x)=x^{+}=\max \{0, x\}$. Then $f$ and $h$ are nonnegative nondecreasing functions. By the convexity of the function $f(x)=x^{+}$, we have

$$
S_{2}^{+}-S_{1}^{+} \geq\left(S_{2}-S_{1}\right) h\left(S_{1}\right)
$$

and then we can get

$$
E\left[\left(S_{2}^{+}-S_{1}^{+}\right) I_{A_{1}}\right] \geq E\left[\left(S_{2}-S_{1}\right) h\left(S_{1}\right) I_{A_{1}}\right] .
$$

Since $h\left(S_{1}\right) I_{A_{1}}$ is a nonnegative nondecreasing function of $S_{1}$ and $\left\{S_{n}, n \geq 1\right\}$ is a demisubmartingale, we have

$$
E\left[\left(S_{2}^{+}-S_{1}^{+}\right) I_{A_{1}}\right] \geq E\left[\left(S_{2}-S_{1}\right) h\left(S_{1}\right) I_{A_{1}}\right] \geq 0 .
$$

So we can get

$$
\begin{aligned}
\varepsilon P(A) & \leq E\left(c_{2} S_{2}^{+} I_{A_{1} \cup A_{2}}\right)+\sum_{j=3}^{n} E\left(c_{j} S_{j}^{+} I_{A_{j}}\right) \\
& =E\left(c_{2} S_{2}^{+} I_{A_{1} \cup A_{2}}\right)+E\left(c_{3} S_{3}^{+} I_{A_{3}}\right)+\sum_{j=4}^{n} E\left(c_{j} S_{j}^{+} I_{A_{j}}\right) .
\end{aligned}
$$


Since $A_{1} \cap A_{2} \cap A_{3}=\emptyset$, one has $I_{A_{3}}=I_{A_{1} \cup A_{2} \cup A_{3}}-I_{A_{1} \cup A_{2}}$. Thus we have

$$
\begin{aligned}
\varepsilon P(A) & \leq E\left(c_{2} S_{2}^{+} I_{A_{1} \cup A_{2}}\right)+E\left[c_{3} S_{3}^{+}\left(I_{A_{1} \cup A_{2} \cup A_{3}}-I_{A_{1} \cup A_{2}}\right)\right]+\sum_{j=4}^{n} E\left(c_{j} S_{j}^{+} I_{A_{j}}\right) \\
& =E\left(c_{3} S_{3}^{+} I_{A_{1} \cup A_{2} \cup A_{3}}\right)+E\left[\left(c_{2} S_{2}^{+}-c_{3} S_{3}^{+}\right) I_{A_{1} \cup A_{2}}\right]+\sum_{j=4}^{n} E\left(c_{j} S_{j}^{+} I_{A_{j}}\right) \\
& \leq E\left(c_{3} S_{3}^{+} I_{A_{1} \cup A_{2} \cup A_{3}}\right)+c_{3} E\left[\left(S_{2}^{+}-S_{3}^{+}\right) I_{A_{1} \cup A_{2}}\right]+\sum_{j=4}^{n} E\left(c_{j} S_{j}^{+} I_{A_{j}}\right) \\
& =E\left(c_{3} S_{3}^{+} I_{A_{1} \cup A_{2} \cup A_{3}}\right)-c_{3} E\left[\left(S_{3}^{+}-S_{2}^{+}\right) I_{A_{1} \cup A_{2}}\right]+\sum_{j=4}^{n} E\left(c_{j} S_{j}^{+} I_{A_{j}}\right) .
\end{aligned}
$$

By the convexity of the function $f(x)=x^{+}$again,

$$
S_{3}^{+}-S_{2}^{+} \geq\left(S_{3}-S_{2}\right) h\left(S_{2}\right)
$$

then

$$
E\left[\left(S_{3}^{+}-S_{2}^{+}\right) I_{A_{1} \cup A_{2}}\right] \geq E\left[\left(S_{3}-S_{2}\right) h\left(S_{2}\right) I_{A_{1} \cup A_{2}}\right] .
$$

Obviously, $A_{1} \cup A_{2}=\left\{\max \left(c_{1} S_{1}, c_{2} S_{2}\right) \geq \varepsilon\right\}$ and $I_{A_{1} \cup A_{2}}$ is a nonnegative and componentwise nondecreasing function of $\left\{S_{1}, S_{2}\right\}$, then $h\left(S_{2}\right) I_{A_{1} \cup A_{2}}$ is a nonnegative and componentwise nondecreasing function of $\left\{S_{1}, S_{2}\right\}$. By the demisubmartingale property, the righthand side of (2.6) is nonnegative. Thus

$$
E\left[\left(S_{3}^{+}-S_{2}^{+}\right) I_{A_{1} \cup A_{2}}\right] \geq 0
$$

and the right-hand side of (2.4) is bounded by

$$
E\left(c_{3} S_{3}^{+} I_{A_{1} \cup A_{2} \cup A_{3}}\right)+\sum_{j=4}^{n} E\left(c_{j} S_{j}^{+} I_{A_{j}}\right) .
$$

Working in this manner we prove that

$$
\begin{aligned}
\varepsilon P(A) & \leq E\left(c_{n-1} S_{n-1}^{+} I_{A_{1} \cup A_{2} \cup \ldots \cup A_{n-1}}\right)+E\left(c_{n} S_{n}^{+} I_{A_{n}}\right) \\
& =E\left(c_{n-1} S_{n-1}^{+} I_{A_{1} \cup A_{2} \cup \cdots \cup A_{n-1}}\right)+E\left[c_{n} S_{n}^{+}\left(I_{A_{1} \cup A_{2} \cup \ldots \cup A_{n}}-I_{A_{1} \cup A_{2} \cup \cdots \cup A_{n-1}}\right)\right] \\
& \leq c_{n} E\left(S_{n}^{+} I_{A}\right)-c_{n} E\left[\left(S_{n}^{+}-S_{n-1}^{+}\right) I_{A_{1} \cup A_{2} \cup \cdots \cup A_{n-1}}\right] .
\end{aligned}
$$

By the convexity of the function $f(x)=x^{+}$, we have

$$
S_{n}^{+}-S_{n-1}^{+} \geq\left(S_{n}-S_{n-1}\right) h\left(S_{n-1}\right) .
$$

Hence

$$
E\left[\left(S_{n}^{+}-S_{n-1}^{+}\right) I_{A_{1} \cup A_{2} \cup \ldots \cup A_{n-1}}\right] \geq E\left[\left(S_{n}-S_{n-1}\right) h\left(S_{n-1}\right) I_{A_{1} \cup A_{2} \cup \ldots \cup A_{n-1}}\right] .
$$


Since $A_{1} \cup A_{2} \cup \cdots \cup A_{n-1}=\left\{\max \left(c_{1} S_{1}, c_{2} S_{2}, \ldots, c_{n-1} S_{n-1}\right) \geq \varepsilon\right\}, I_{A_{1} \cup A_{2} \cup \cdots \cup A_{n-1}}$ is a nonnegative and componentwise nondecreasing function of $\left\{S_{1}, S_{2}, \ldots, S_{n-1}\right\}$. Then $h\left(S_{n-1}\right) \times$ $I_{A_{1} \cup A_{2} \cup \ldots \cup A_{n-1}}$ is a nonnegative and componentwise nondecreasing function of $\left\{S_{1}, S_{2}\right.$, $\left.\ldots, S_{n-1}\right\}$. As $\left\{S_{n}, n \geq 1\right\}$ forms a demisubmartingale and $\left\{c_{n}, n \geq 1\right\}$ is a sequence of positive numbers, we have

$$
c_{n} E\left[\left(S_{n}-S_{n-1}\right) h\left(S_{n-1}\right) I_{A_{1} \cup A_{2} \cup \ldots \cup A_{n-1}}\right] \geq 0 .
$$

Consequently, it follows from (2.7), (2.8) and (2.9) that

$$
\varepsilon P(A) \leq E\left(c_{n} S_{n}^{+} I_{A}\right)
$$

So (2.1) is proved.

Corollary 2.1 Assume that $\left\{S_{n}, n \geq 1\right\}$ is a demisubmartingale or a demimartingale with $S_{0}=0$. Let $g$ be a nondecreasing convex function such that $g\left(S_{n}\right) \in L^{1}, n \geq 1$ and $\left\{c_{n}, n \geq 1\right\}$ be a nondecreasing sequence of positive numbers. Then, for any $\varepsilon>0$,

$$
\varepsilon P\left\{\max _{1 \leq k \leq n} c_{k} g\left(S_{k}\right) \geq \varepsilon\right\} \leq E\left[c_{n} g^{+}\left(S_{n}\right) I\left(\max _{1 \leq k \leq n} c_{k} g\left(S_{k}\right) \geq \varepsilon\right)\right] .
$$

Proof By Lemma 1.1, $\left\{g\left(S_{n}\right), n \geq 1\right\}$ is a demisubmartingale. By Theorem 2.1, we obtain the result of (2.10).

Remark 2.1 Chow [25] proved a maximal inequality for submartingales, which contains the Hajek-Renyi inequality and other inequalities as special cases (see Theorem 1 of Chow [25]). Christofides [4] generalized Theorem 1 of Chow [25] and obtained a Chow type maximal inequality for demimartingales (see Theorem 2.1 of Christofides [4]). Wang [7] generalized Theorem 2.1 of Christofides [4] to the nonnegative convex functions (see Theorem 2.1 of Wang [7]). Based on Christofides [4] and Wang [7], Wang and $\mathrm{Hu}$ [9] obtained some similar maximal inequalities for demisubmartingales and demimartingales (see Theorem 2.1 and Theorem 2.2 of Wang and $\mathrm{Hu}$ [9]). Inspired by these papers, we also get some similar Chow type maximal inequality for demisubmartingales and demimartingales (see Theorem 2.1 and Corollary 2.1).

Second, we provide a minimal type inequalities for a sequence of nonnegative demimartingales.

Theorem 2.2 Let $\left\{S_{n}, n \geq 1\right\}$ be a nonnegative demimartingale with $S_{0}=0$ and $\left\{c_{n}, n \geq 1\right\}$ be a nonincreasing sequence of positive numbers. Then, for any $\varepsilon>0$,

$$
\varepsilon P\left\{\min _{1 \leq k \leq n} c_{k} S_{k} \leq \varepsilon\right\} \geq c_{n} E\left[S_{n} I\left(\min _{1 \leq k \leq n} c_{k} S_{k} \leq \varepsilon\right)\right]
$$

Proof Following Christofides [4], we let $A=\left\{\min _{1 \leq k \leq n} c_{k} S_{k} \leq \varepsilon\right\}, n \geq 1$. Then $A$ can be written as $A=\bigcup_{j=1}^{n} A_{j}$, where $A_{1}=\left\{c_{1} S_{1} \leq \varepsilon\right\}, A_{j}=\left\{c_{i} S_{i}>\varepsilon, 1 \leq i<j, c_{j} S_{j} \leq \varepsilon\right\}, 1<j \leq n$, and 
$A_{i} \cap A_{j}=\emptyset$ when $i \neq j$. Thus, similar to the proof of (2.2),

$$
\begin{aligned}
\varepsilon P(A) & =\varepsilon \sum_{j=1}^{n} P\left(A_{j}\right)=\sum_{j=1}^{n} E\left(\varepsilon I_{A_{j}}\right) \geq \sum_{j=1}^{n} E\left(c_{j} S_{j} I_{A_{j}}\right) \\
& =E\left(c_{1} S_{1} I_{A_{1}}\right)+E\left(c_{2} S_{2} I_{A_{2}}\right)+\sum_{j=3}^{n} E\left(c_{j} S_{j} I_{A_{j}}\right) \\
& =E\left(c_{1} S_{1} I_{A_{1}}\right)+E\left[c_{2} S_{2}\left(I_{A_{1} \cup A_{2}}-I_{A_{1}}\right)\right]+\sum_{j=3}^{n} E\left(c_{j} S_{j} I_{A_{j}}\right) \\
& =E\left(c_{2} S_{2} I_{A_{1} \cup A_{2}}\right)+E\left[\left(c_{1} S_{1}-c_{2} S_{2}\right) I_{A_{1}}\right]+\sum_{j=3}^{n} E\left(c_{j} S_{j} I_{A_{j}}\right) \\
& \geq E\left(c_{2} S_{2} I_{A_{1} \cup A_{2}}\right)+c_{2} E\left[\left(S_{1}-S_{2}\right) I_{A_{1}}\right]+\sum_{j=3}^{n} E\left(c_{j} S_{j} I_{A_{j}}\right) \\
& =E\left(c_{2} S_{2} I_{A_{1} \cup A_{2}}\right)+c_{2} E\left[\left(S_{2}-S_{1}\right)\left(-I_{A_{1}}\right)\right]+\sum_{j=3}^{n} E\left(c_{j} S_{j} I_{A_{j}}\right),
\end{aligned}
$$

which is from the fact that $A_{1} \cap A_{2}=\emptyset$ and $I_{A_{2}}=I_{A_{1} \cup A_{2}}-I_{A_{1}}$. Since $I_{A_{1}}$ is a nonincreasing function of $S_{1},-I_{A_{1}}$ is a nondecreasing function of $S_{1}$. By the definition of a demimartingale, one has

$$
E\left[\left(S_{2}-S_{1}\right)\left(-I_{A_{1}}\right)\right] \geq 0
$$

So we can get

$$
\begin{aligned}
\varepsilon P(A) & \geq E\left(c_{2} S_{2} I_{A_{1} \cup A_{2}}\right)+\sum_{j=3}^{n} E\left(c_{j} S_{j} I_{A_{j}}\right) \\
& =E\left(c_{2} S_{2} I_{A_{1} \cup A_{2}}\right)+E\left(c_{3} S_{3} I_{A_{3}}\right)+\sum_{j=4}^{n} E\left(c_{j} S_{j} I_{A_{j}}\right) \\
& =E\left(c_{2} S_{2} I_{A_{1} \cup A_{2}}\right)+E\left[c_{3} S_{3}\left(I_{A_{1} \cup A_{2} \cup A_{3}}-I_{A_{1} \cup A_{2}}\right)\right]+\sum_{j=4}^{n} E\left(c_{j} S_{j} I_{A_{j}}\right) \\
& =E\left(c_{3} S_{3} I_{A_{1} \cup A_{2} \cup A_{3}}\right)+E\left[\left(c_{2} S_{2}-c_{3} S_{3}\right) I_{A_{1} \cup A_{2}}\right]+\sum_{j=4}^{n} E\left(c_{j} S_{j} I_{A_{j}}\right) \\
& \geq E\left(c_{3} S_{3} I_{A_{1} \cup A_{2} \cup A_{3}}\right)+c_{3} E\left[\left(S_{2}-S_{3}\right) I_{A_{1} \cup A_{2}}\right]+\sum_{j=4}^{n} E\left(c_{j} S_{j} I_{A_{j}}\right) \\
& =E\left(c_{3} S_{3} I_{A_{1} \cup A_{2} \cup A_{3}}\right)+c_{3} E\left[\left(S_{3}-S_{2}\right)\left(-I_{A_{1} \cup A_{2}}\right)\right]+\sum_{j=4}^{n} E\left(c_{j} S_{j} I_{A_{j}}\right) .
\end{aligned}
$$

Since $A_{1} \cup A_{2}=\left\{\min \left(c_{1} S_{1}, c_{2} S_{2}\right) \leq \varepsilon\right\}, I_{A_{1} \cup A_{2}}$ is a componentwise nonincreasing function of $\left\{S_{1}, S_{2}\right\}$ and $-I_{A_{1} \cup A_{2}}$ is a componentwise nondecreasing function of $\left\{S_{1}, S_{2}\right\}$. By the 
definition of a demimartingale,

$$
E\left[\left(S_{3}-S_{2}\right)\left(-I_{A_{1} \cup A_{2}}\right)\right] \geq 0 .
$$

It follows from (2.13) and (2.14) that

$$
\varepsilon P(A) \geq E\left(c_{3} S_{3} I_{A_{1} \cup A_{2} \cup A_{3}}\right)+\sum_{j=4}^{n} E\left(c_{j} S_{j} I_{A_{j}}\right)
$$

By iterations,

$$
\begin{aligned}
\varepsilon P(A) & \geq E\left(c_{n-1} S_{n-1} I_{A_{1} \cup A_{2} \cup \ldots \cup A_{n-1}}\right)+E\left(c_{n} S_{n} I_{A_{n}}\right) . \\
& =E\left(c_{n-1} S_{n-1} I_{A_{1} \cup A_{2} \cup \ldots \cup A_{n-1}}\right)+E\left[c_{n} S_{n}\left(I_{A_{1} \cup A_{2} \cup \ldots \cup A_{n}}-I_{A_{1} \cup A_{2} \cup \ldots \cup A_{n-1}}\right)\right] \\
& \geq c_{n} E\left(S_{n} I_{A}\right)+c_{n} E\left[\left(S_{n}-S_{n-1}\right)\left(-I_{A_{1} \cup A_{2} \cup \ldots \cup A_{n-1}}\right)\right] .
\end{aligned}
$$

Since $A_{1} \cup A_{2} \cup \cdots \cup A_{n-1}=\left\{\min \left(c_{1} S_{1}, c_{2} S_{2}, \ldots, c_{n-1} S_{n-1}\right) \leq \varepsilon\right\}, I_{A_{1} \cup A_{2} \cup \cdots \cup A_{n-1}}$ is a componentwise nonincreasing function of $\left\{S_{1}, S_{2}, \ldots, S_{n-1}\right\}$ and $-I_{A_{1} \cup A_{2} \cup \ldots \cup A_{n-1}}$ is a componentwise nondecreasing function of $\left\{S_{1}, S_{2}, \ldots, S_{n-1}\right\}$. By the fact that $\left\{S_{n}, n \geq 1\right\}$ is a nonnegative demimartingale and $\left\{c_{k}, k \geq 1\right\}$ is a nonincreasing sequence of positive numbers, it is checked that

$$
c_{n} E\left[\left(S_{n}-S_{n-1}\right)\left(-I_{A_{1} \cup A_{2} \cup \ldots \cup A_{n-1}}\right)\right] \geq 0 .
$$

Finally, by (2.15) and (2.16), we get

$$
\varepsilon P(A) \geq c_{n} E\left(S_{n} I_{A}\right) .
$$

So (2.11) holds.

Corollary 2.2 Let $\left\{S_{n}, n \geq 1\right\}$ be a demimartingale. Then, for any $\varepsilon>0$,

$$
\varepsilon P\left\{\min _{1 \leq k \leq n} S_{k} \leq \varepsilon\right\} \geq \int_{\left\{\min _{1 \leq k \leq n} S_{k} \leq \varepsilon\right\}} S_{n} d P .
$$

Proof By the proof of Theorem 2.2 with $c_{k} \equiv 1$, we can get the minimal inequality for demimartingales without the assumption of nonnegativeness.

Remark 2.2 Newman and Wright [1] obtained some inequalities for demisubmartingales and demimartingales, including maximal and minimal inequalities (see Theorem 3 of Newman and Wright [1]). Prakasa Rao [8] generalized some results of Newman and Wright [1] and got minimal type inequalities for demisubmartingales (see Theorems 2.82.10 of Prakasa Rao [8]). Wang et al. [11] also obtained some minimal inequalities for nonnegative demimartingales (see Theorem 2.1, Corollary 2.1 and Corollary 2.2 of Wang et al. [11]). Similar to Theorem 2.8 of Prakasa Rao [8] and Theorem 2.1 of Wang et al. [11], we get some minimal type inequalities for nonnegative demimartingales in Theorem 2.2 and Corollary 2.3. It is pointed out that Corollary 2.2 is not a new result (see Theorem 2.9 of Prakasa Rao [8], Corollary 2.1 of Hu et al. [17], Corollary 2.1 of Wang et al. [11]). 
Third, we consider the Chow type maximal inequality for $N$-demimartingales. Similar to Chow [25] and Christofides [4], Prakasa Rao [15] obtained a Chow type maximal inequality for $N$-demimartingales.

Theorem 2.3 (see Theorem 3.1 of Prakasa Rao [15] or Theorem 3.5.1 of Prakasa Rao [21]) Assume that $\left\{S_{n}, n \geq 1\right\}$ is an $N$-demimartingale with $S_{0}=0$ and $m(\cdot)$ is a nonnegative nondecreasing function on $\mathbb{R}$ with $m(0)=0$. Let $g(\cdot)$ be a function on $\mathbb{R}$ with $g(0)=0$ and suppose that

$$
g(x)-g(y) \geq(y-x) h(y)
$$

for all $x, y$, where $h(\cdot)$ is a nonnegative and nondecreasing function. Further assume that $\left\{c_{k}, 1 \leq k \leq n\right\}$ is a sequence of positive numbers such that $\left(c_{k}-c_{k+1}\right) g\left(S_{k}\right) \geq 0$ for $1 \leq k \leq$ $n-1$. Define $Y_{k}=\max _{1 \leq j \leq k} c_{j} g\left(S_{j}\right), k \geq 1, Y_{0}=0$. Then

$$
E\left(\int_{0}^{Y_{n}} u d m(u)\right) \leq \sum_{i=1}^{n} c_{i} E\left[\left(g\left(S_{i}\right)-g\left(S_{i-1}\right)\right) m\left(Y_{n}\right)\right]
$$

Let $\varepsilon>0$ and define $m(t)=1$ if $t \geq \varepsilon$ and $m(t)=0$ if $t<\varepsilon$. By Theorem 2.3,

$$
\varepsilon P\left(Y_{n} \geq \varepsilon\right) \leq \sum_{i=1}^{n} c_{i} E\left[\left(g\left(S_{i}\right)-g\left(S_{i-1}\right)\right) I\left(Y_{n} \geq \varepsilon\right)\right]
$$

(see (3.5.10) of Prakasa Rao [21]) was obtained. It can be seen that $g(x)=-\alpha x, \alpha \geq 0$, and $g(x)=-\alpha x^{+}, \alpha \geq 0$, satisfy the condition of (2.17) (see Prakasa Rao $[15,21]$ ).

It is a fact that if $\left\{S_{n}\right\}_{n \geq 1}$ is an $N$-demimartingale, then $\left\{-S_{n}\right\}_{n \geq 1}$ is also an $N$-demimartingale (see Christofides [2] or Prakasa Rao [21]). By using Theorem 2.3, Hadjikyriakou [22] got the following maximal inequality for $N$-demimartingales.

Corollary 2.3 (Hadjikyriakou [22, Theorem 3.2.1]) Assume that $\left\{S_{n}, n \geq 1\right\}$ is an $N$ demimartingale. Then, for every $\varepsilon>0$,

$$
\varepsilon P\left(\max _{1 \leq k \leq n} S_{k} \geq \varepsilon\right) \leq E\left(S_{n} I\left(\max _{1 \leq k \leq n} S_{k} \geq \varepsilon\right)\right)
$$

But we find that the Chow type maximal inequality for $N$-demimartingales, i.e., Theorem 2.3, is not true. We give an example as follows.

An example for $N$-demimartingales Let $g(x)=-x, m(x)=x^{+}, c_{1}=c_{2}=1, S_{0}=0$. Assume that $S_{1}$ and $S_{2}$ are independent random variables with probability distributions

$$
S_{1} \sim\left(\begin{array}{cc}
-2 & 2 \\
\frac{1}{2} & \frac{1}{2}
\end{array}\right), \quad S_{2} \sim\left(\begin{array}{cc}
-1 & 1 \\
\frac{1}{2} & \frac{1}{2}
\end{array}\right)
$$

In addition, let $Y_{1}=c_{1} g\left(S_{1}\right)=-S_{1}$ and

$$
Y_{2}=\max \left\{c_{1} g\left(S_{1}\right), c_{2} g\left(S_{2}\right)\right\}=\max \left\{-S_{1},-S_{2}\right\} .
$$


It is easy to check that for any nondecreasing function $f$,

$$
\begin{aligned}
E[ & \left.\left(S_{2}-S_{1}\right) f\left(S_{1}\right)\right] \\
= & {[-1-(-2)] f(-2) \times \frac{1}{4}+(-1-2) f(2) \times \frac{1}{4}+[1-(-2)] f(-2) } \\
& \times \frac{1}{4}+(1-2) f(2) \times \frac{1}{4} \\
= & f(-2)-f(2) \leq 0 .
\end{aligned}
$$

Hence $\left\{S_{1}, S_{2}\right\}$ is an $N$-demimartingale. It follows from the distribution of $S_{1}$ that

$$
Y_{1} \sim\left(\begin{array}{cc}
-2 & 2 \\
\frac{1}{2} & \frac{1}{2}
\end{array}\right)
$$

Meanwhile,

$$
\begin{aligned}
& P\left(Y_{2}=1\right)=P\left(S_{1}=2, S_{2}=-1\right)=\frac{1}{4}, \\
& P\left(Y_{2}=-1\right)=P\left(S_{1}=2, S_{2}=1\right)=\frac{1}{4}, \\
& P\left(Y_{2}=2\right)=P\left(S_{1}=-2, S_{2}=1\right)+P\left(S_{1}=-2, S_{2}=-1\right)=\frac{1}{4}+\frac{1}{4}=\frac{1}{2},
\end{aligned}
$$

SO

$$
Y_{2} \sim\left(\begin{array}{ccc}
-1 & 1 & 2 \\
\frac{1}{4} & \frac{1}{4} & \frac{1}{2}
\end{array}\right)
$$

It can be seen that if $Y_{2}(\omega) \geq 0$, then

$$
\int_{0}^{Y_{2}} u d m(u)=\int_{0}^{Y_{2}} u d u=\frac{1}{2} Y_{2}^{2} .
$$

Otherwise, for the case $Y_{2}(\omega)<0$, one has

$$
\int_{0}^{Y_{2}} u d m(u)=0
$$

Consequently,

$$
\int_{0}^{Y_{2}} u d m(u)=\frac{1}{2} Y_{2}^{2} I\left(Y_{2} \geq 0\right) \sim\left(\begin{array}{ccc}
0 & \frac{1}{2} & 2 \\
\frac{1}{4} & \frac{1}{4} & \frac{1}{2}
\end{array}\right) .
$$

On the other hand, we can calculate that

$$
E\left[\int_{0}^{Y_{2}} u d m(u)\right]=\frac{9}{8}
$$


and

$$
\begin{aligned}
\sum_{k=1}^{2} c_{k} E\left\{\left[g\left(S_{k}\right)-g\left(S_{k-1}\right)\right] m\left(Y_{2}\right)\right\} & =E\left[g\left(S_{2}\right) m\left(Y_{2}\right)\right]=-E\left(S_{2} Y_{2}^{+}\right) \\
& =-\left[-1 \times 1 \times \frac{1}{4}+1 \times 2 \times \frac{1}{4}+(-1) \times 2 \times \frac{1}{4}\right] \\
& =\frac{1}{4} .
\end{aligned}
$$

But we have

$$
\frac{9}{8}=E\left[\int_{0}^{Y_{2}} u d m(u)\right]>\sum_{k=1}^{2} c_{k} E\left\{\left[g\left(S_{k}\right)-g\left(S_{k-1}\right)\right] m\left(Y_{2}\right)\right\}=\frac{1}{4},
$$

which is contrary to (2.18). Therefore, Theorem 2.3 is not true. In fact, in the proof of Theorem 3.1 of Prakasa Rao [15] or the proof of Theorem 3.5.1 of Prakasa Rao [8], it was given that $h\left(S_{i}\right) m\left(Y_{i}\right)$ is a nondecreasing function of $S_{1}, S_{2}, \ldots, S_{i}$. But by checking the proof carefully, we find that one cannot find that $h\left(S_{i}\right) m\left(Y_{i}\right)$ is a nondecreasing function of $S_{1}, S_{2}, \ldots, S_{i}$ under the conditions of Theorem 2.3.

Similarly, it can be checked that

$$
P\left(Y_{2} \geq 1\right)=\frac{1}{2}+\frac{1}{4}=\frac{3}{4},
$$

and

$$
\begin{aligned}
\sum_{k=1}^{2} E\left[\left(g\left(S_{k}\right)-g\left(S_{k-1}\right)\right) I\left(Y_{2} \geq 1\right)\right] & =-E\left[S_{2} I\left(Y_{2} \geq 1\right)\right] \\
& =-\left[-1 \times 1 \times \frac{1}{4}+1 \times 1 \times \frac{1}{4}+(-1) \times 1 \times \frac{1}{4}\right] \\
& =\frac{1}{4} .
\end{aligned}
$$

Then

$$
P\left(Y_{2} \geq 1\right)>-E\left[S_{2} I\left(Y_{2} \geq 1\right)\right]
$$

which is contrary to (2.19). So (2.19) is not true.

Meanwhile, one has

$$
P\left(\max _{1 \leq k \leq 2} S_{k} \geq 1\right)=P\left(S_{1}=2, S_{2}=-1\right)+P\left(S_{1}=2, S_{2}=1\right)+P\left(S_{1}=-2, S_{2}=1\right)=\frac{3}{4}
$$

and

$$
E\left[S_{2} I\left(\max \left(S_{1}, S_{2}\right)\right) \geq 1\right]=-1 \times \frac{1}{4}+1 \times \frac{1}{4}+1 \times \frac{1}{4}=\frac{1}{4} .
$$

So

$$
\frac{3}{4}=P\left(\max _{1 \leq k \leq 2} S_{k} \geq 1\right)>E\left[S_{2} I\left(\max \left(S_{1}, S_{2}\right)\right) \geq 1\right]=\frac{1}{4},
$$


which is contrary to (2.20). Thus, (2.20) is not true. There are some problems of maximal type inequalities for $N$-demimartingales in the literature such as Wang et al. [19], Hu et al. [13], Wang et al. [14] and Yang and $\mathrm{Hu}$ [20]. It is interesting to investigate the maximal type inequalities of $N$-demimartingales for researchers in the future.

\section{Competing interests}

The authors declare that they have no competing interests.

\section{Authors' contributions}

All authors read and approved the final manuscript.

\section{Acknowledgements}

The authors are deeply grateful to the editor and anonymous referees whose insightful comments and suggestions have contributed substantially to the improvement of this paper. This work is supported by the National Natural Science Foundation of China (11171001, 11201001, 11326172), Natural Science Foundation of Anhui Province (1408085QA02), Introduction Projects of Academic and Technology Leaders of Anhui University, Key Program of Research and Development Foundation of Hefei University (13KY05ZD), Key NSF of Anhui Educational Committee (KJ2014A255), Program of Student Science Research Training of Anhui University (KYXL2014015) and Doctoral Research Start-up Funds Projects of Anhui University.

Received: 11 July 2014 Accepted: 12 November 2014 Published: 12 Dec 2014

\section{References}

1. Newman, CM, Wright, AL: Associated random variables and martingale inequalities. Z. Wahrscheinlichkeitstheor. Verw. Geb. 59(3), 361-371 (1982)

2. Christofides, TC: Maximal inequalities for N-demimartingales. Arch. Inequal. Appl. 50(1), 397-408 (2003)

3. Wood, TE: Sample paths of demimartingales. In: Probability Theory on Vector Spaces III. Lecture Notes in Mathematics, vol. 1080, pp. 365-373. Springer, New York (1984). doi:10.1007/BFb0099807

4. Christofides, TC: Maximal inequalities for demimartingales and a strong law of large numbers. Stat. Probab. Lett. 50(4), 357-363 (2000)

5. Prakasa Rao, BLS: Whittle type inequality for demisubmartingales. Proc. Am. Math. Soc. 130(12), 3719-3724 (2002)

6. Christofides, TC: U-Statistics on associated random variables. J. Stat. Plan. Inference 119(1), 1-15 (2004)

7. Wang, JF: Maximal inequalities for associated random variables and demimartingales. Stat. Probab. Lett. 66(3), 347-354 (2004)

8. Prakasa Rao, BLS: On some maximal inequalities for demisubmartingales and N-demisupermartingales. J. Inequal. Pure Appl. Math. 8(4), 112 (2007)

9. Wang, XJ, Hu, SH: Maximal inequalities for demimartingales and their applications. Sci. China Ser. A 52(10), 2207-2217 (2009)

10. Wang, XJ, Hu, SH, Zhao, T, Yang, WZ: Doob's type inequality and strong law of large numbers for demimartingales J. Inequal. Appl. 2010, Article ID 838301 (2010)

11. Wang, XJ, Hu, SH, Yang, WZ, Shen, Y: Some new results for demimartingales. Appl. Math. J. Chin. Univ. Ser. B 26(1), 14-22 (2011)

12. Christofides, TC, Hadjikyriakou, M: Maximal and moment inequalities for demimartingales and $\mathrm{N}$-demimartingales. Stat. Probab. Lett. 82(3), 683-691 (2012)

13. $\mathrm{Hu}, \mathrm{SH}$, Wang, $\mathrm{XH}$, Yang, WZ, Wang, $\mathrm{XJ}$ : Some inequalities for demimartingales and $\mathrm{N}$-demimartingales. Stat. Probab. Lett. 82(2), 232-239 (2012)

14. Wang, XJ, Prakasa Rao, BLS, Hu, SH, Yang, WZ: On some maximal inequalities for demimartingales and $\mathrm{N}$-demimartingales based on concave Young functions. J. Math. Anal. Appl. 396(2), 434-440 (2012)

15. Prakasa Rao, BLS: On some inequalities for N-demimartingales. J. Indian Soc. Agric. Stat. 57, 208-216 (2004)

16. Christofides, TC, Hadjikyriakou, M: Exponential inequalities for $\mathrm{N}$-demimartingales and negatively associated random variables. Stat. Probab. Lett. 79(19), 2060-2065 (2009)

17. Hu, SH, Yang, WZ, Wang, XJ, Shen, Y: A note on the inequalities for N-demimartingales and demimartingales. J. Syst. Sci. Math. Sci. 30(8), 1052-1058 (2010)

18. Hadjikyriakou, M: Marcinkiewicz-Zygmund inequality for nonnegative $N$-demimartingales and related results. Stat. Probab. Lett. 81(6), 678-684 (2011)

19. Wang, XJ, Hu, SH, Prakasa Rao, BLS, Yang, WZ: Maximal inequalities for $\mathrm{N}$-demimartingale and strong law of large numbers. Stat. Probab. Lett. 81(9), 1348-1353 (2011)

20. Yang, WZ, Hu, SH: Some maximal type inequalities for $\mathrm{N}$-demimartingales and related results. Math. Inequal. Appl. 17(2), 731-747 (2014)

21. Prakasa Rao, BLS: Associated Sequences, Demimartingales and Nonparametric Inference. Springer, Basel (2012)

22. Hadjikyriakou, M: Probability and moment inequalities for demimartingales and associated random variables. Ph.D. dissertation, Department of Mathematics and Statistics, University of Cyprus, Nicosia (2010)

23. Christofides, TC, Hadjikyriakou, M: Conditional demimartingales and related results. J. Math. Anal. Appl. 398(1), 380-391 (2013)

24. Wang, XH, Wang, XJ: Some inequalities for conditional demimartingales and conditional N-demimartingales. Stat. Probab. Lett. 83(3), 700-709 (2013)

25. Chow, YS: A martingale inequality and the law of large numbers. Proc. Am. Math. Soc. 11(1), 107-111 (1960) 
10.1186/1029-242X-2014-489

Cite this article as: Dai et al.: Some results for demimartingales and $\mathrm{N}$-demimartingales. Journal of Inequalities and Applications 2014, 2014:489

Submit your manuscript to a SpringerOpen ${ }^{\circ}$ journal and benefit from:

- Convenient online submission

- Rigorous peer review

- Immediate publication on acceptance

- Open access: articles freely available online

- High visibility within the field

- Retaining the copyright to your article

Submit your next manuscript at $\gg$ springeropen.com 